\title{
Life-threatening cardiotoxicity due to chronic oral phenytoin overdose
}

\author{
Chih-M in Su, Chia-Te Kung, Yu-Chin W ang ${ }^{1}$, Cheng-H sien Lü \\ Departments of Emergency Medicine, ${ }^{1}$ Pharmacy, and ${ }^{2}$ Neurology, Chang Gung Memorial Hospital-Kaohsiung Medical Center, \\ Chang Gung University College of Medicine, Kaobsiung, Taiwan
}

\begin{abstract}
Severe cardiac adverse effects are often related to intravenous phenytoin overdose. However, there is no reported cardiotoxicity resulting from oral overdose of phenytoin. We report a patient with post-traumatic epilepsy who received oral phenytoin for five months and developed life-threatening junctional bradycardia, with his serum phenytoin level reaching up to $91 \mu \mathrm{g} / \mathrm{mL}$. The patient was successfully treated with temporary transvenous pacemaker implantation for his severe bradycardia and hypotension. To our knowledge, our patient had the most serious cardiovascular toxicity ever reported with chronic oral phenytoin overdose. From emergency department (ED) physician's perspective, when a patient with dysrhythmias and cardiovascular collapse is presented to the ED, severe phenytoin overdose should be considered in patients on oral phenytoin with hyperbilirubinemia, hypoalbuminemia, and severe electrolyte imbalance.
\end{abstract}

Address for correspondence: Prof. Cheng-Hsien Lu, Department of Neurology, Chang Gung Memorial Hospital 123, Ta Pei Road, Niao Sung Hsiang Kaohsiung Hsien, Taiwan.

E-mail: chlu99@ms44.url.com.tw

DOI: $10.4103 / 0028-3886.51296$

Key words: $\mathrm{C}$ ardio toxicity, oral phenyto in, overdose

\section{Introduction}

Phenytoin is one of the most commonly used antiepileptic drugs (AED). However it has a narrow therapeutic range, requiring therapeutic drug monitoring to assess compliance and evaluate the response to therapy. ${ }^{[1]}$ Toxicity produces predominantly neurologic dysfunction. Reported severe cardiac adverse effects of phenytoin overdose are usually related to intravenous administration. ${ }^{[2,3]}$ Oral phenytoin overdoses in adults are rarely life threatening and seldom give rise to neurologic complications resulting in fatalities. ${ }^{[4-7]}$ We report a hemodynamically unstable patient who presented to the emergency department (ED) with severe junctional bradycardia related to a oral phenytoin overdose.

\section{Case Report}

A 35-year-old male with a history of traumatic intracranial hemorrhage complicated by post-traumatic epilepsy and vegetative state was brought to our ED from a nursing home for bradycardia and low blood pressure. Apart from seizures he was diagnosed to have pulmonary tuberculosis and was on antituberculous medication for the last four months. The patient had been placed on phenytoin $200 \mathrm{mg}$ twice a day for recurrent seizures about 5 months before his current presentation.

On arrival, the patient's vital signs were: Body temperature $36.2^{\circ} \mathrm{C}$, blood pressure $87 / 75 \mathrm{mmHg}$, pulse 35 per minute, and respiratory rate 20 breaths per minute. Random blood glucose was $144 \mathrm{mg} / \mathrm{dL}$. Physical examinations revealed a chronic bedridden man with limb contracture and vegetative status. There was withdrawal to deep pain. Mucous membranes were dry. The electrocardiogram (ECG) revealed a junctional bradycardia with a rate of 35 per minute [Figure 1]. Laboratory evaluation revealed a normal blood count (CBC), creatinine, magnesium, calcium, and troponin- 1 levels. His other laboratory data was: Sodium level $121 \mathrm{mEq} / \mathrm{L}$, potassium $5.4 \mathrm{mEq} / \mathrm{L}$, aspartate aminotransferase $140 \mathrm{U} / \mathrm{L}$, alanine aminotransferase $113 \mathrm{U} / \mathrm{L}$, total bilirubin $2.9 \mathrm{mg} / \mathrm{dL}$, and albumin $2.3 \mathrm{~g} / \mathrm{dL}$. The initial phenytoin level was $91 \mu \mathrm{g} / \mathrm{mL}$.

A temporary transcutaneous pacemaker was initially implanted when fluid resuscitation, intravenous atropine (1 mg) and dopamine infusion failed to correct blood pressure and junctional bradycardia. Three hours later, 


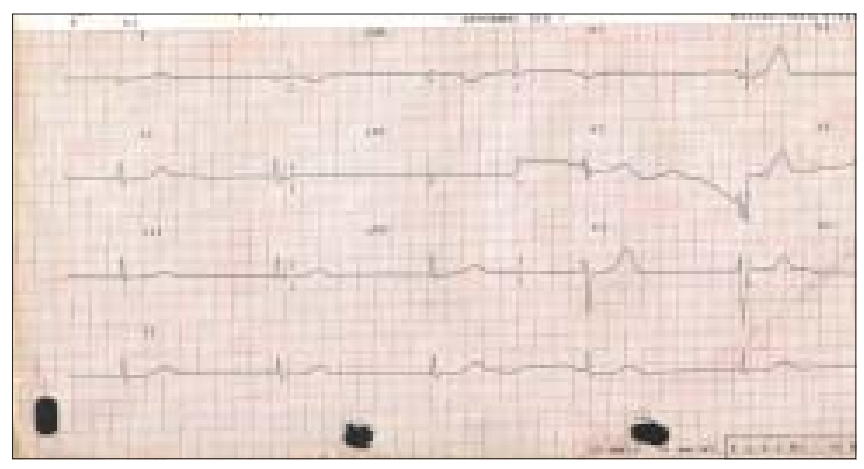

Figure 1: The electrocardiogram (ECG) revealed a junctional bradycardia at a rate of $35 \mathrm{bpm}$

in a second attempt, a temporary transvenous pacemaker was implanted. The patient was admitted to the intensive care unit. With potassium-removing resin his potassium level decreased to $4.8 \mathrm{mEq} / \mathrm{L}$ and sodium level reached $127 \mathrm{mEq} / \mathrm{L}$ after $0.9 \%$ normal saline infusion over 10 hours. Follow-up monitoring of phenytoin levels showed gradual decline [Table 1]. ECG improved to normal sinus rhythm three days later, when the patient's phenytoin level decreased to $67.3 \mu \mathrm{g} / \mathrm{mL}$ and no more bradycardia was noted. Anti-TB medications were discontinued on admission and the bilirubin level improved to $1.6 \mathrm{mg} / \mathrm{dL}$ before discharge. The pacemaker was removed, and the patient was discharged 14 days later in the same baseline vegetative state.

\section{Discussion}

The toxic effects of phenytoin depend on the route and duration of exposure, the dosage, as well as genetic mutations in the cytochrome P450 (CYP) enzyme. ${ }^{[8]}$ Intravenous phenytoin can cause cardiac arrhythmias, hypotension, and cardiovascular collapse even when infused at the recommended rate. ${ }^{[2,4]}$ Hypotension often results from a combination of factors: Direct myocardial depression, reduction in cardiac output and peripheral vasodilation. ${ }^{[7]}$

Hyponatremia may play an important role in the development of bradycardia, however, isolated hyponatremia does not result in consistent effects on the patient's ECG. ${ }^{[7]}$ Phenytoin can inhibit cardiac sodium channels $s^{[7,-10]}$ and inhibit phase 0 inward intracellular sodium currents which results in slowing of conduction and widening of the QRS complex. This indicates hyponatremia may contribute to the effect on conduction disturbance or abnormal automaticity with severe phenytoin overdose. Patients with impaired or decreased protein-binding capacity can develop symptoms at total phenytoin concentrations within the therapeutic range.${ }^{[1]}$ Our patient in addition had hyperbilirubinemia and hypoalbuminemia which might be contributing

\begin{tabular}{|c|c|c|c|c|c|c|}
\hline Day & 0 & 3 & 6 & 9 & 11 & 13 \\
\hline Serum phenytoin level $(\mu \mathrm{g} / \mathrm{mL})$ & 91 & 67.3 & 59.5 & 36.8 & 21.3 & 2.65 \\
\hline Elimination rate ( $\mathrm{mg} / \mathrm{kg} /$ day) & 12.86 & 12.03 & 11.77 & 11.03 & 8.47 & 1.96 \\
\hline
\end{tabular}

factors for the phenytoin toxicity. In addition he was also on anti-TB agents, drug interaction is another important factor that can enhance the toxicity of phenytoin. ${ }^{[1]}$ Several of these mechanisms might have contributed for the development of cardiac arrhythmia in our case. The improvements in arrhythmia in our case coincided with the improvement of metabolic derangement and discontinued anti-TB medications.

Phenytoin overdoses are usually detected early when patients develop ataxic gaits, nystagmus, and mental status changes. However, our patient was in a vegetative status, therefore, it is difficult to detect these changes. As a result, his caregiver continued his phenytoin regimen until he was brought to our ED with low blood pressure and severe bradycardia. One study had demonstrated that 44 patients admitted with oral phenytoin overdose with serum concentrations as high as $75 \mu \mathrm{g} / \mathrm{mL}$ did not experience any significant cardiovascular arrhythmias or complications. ${ }^{[11]}$ Phenytoin level in our case was higher than the reported series of patients with oral phenytoin toxicity, which may explain the arrhythmia. Charcoal hemoperfusion is reported to be effective in cases when serial serum levels are increasing or persistently elevated. ${ }^{[12]}$ In our case, charcoal hemoperfusion was at high risk because of the patient's unstable hemodynamic status. Implantation of a temporary transvenous pacemaker is the standard treatment as for the Advanced Cardiovascular Life Support (ACLS) guidelines for severe junctional bradycardia in the ED and it successfully achieved hemodynamic stability before his ECG confirmed a normal sinus rhythm.

This case may remind clinicians that severe cardiotoxicity due to chronic oral phenytoin overdose is possible when the serum phenytoin accumulates to an extremely high level and presence of predisposing factors that can enhance the toxicity.

\section{References}

1. Doyon S. Anticovulsants. In: Goldfrank LR, Flomenbaum NE, Lewin NA, et al. eds. Goldfrank's toxicologic emergencies. $8^{\text {th }}$ ed. New York: McGraw-Hill; 2006, p. 733-5.

2. York RC, Coleridge ST. Cardiopulmonary arrest following intravenous phenytoin loading. Am J Emerg Med 1988;6:255-9.

3. Goldschlager AW, Karliner JS. Ventricular standstill after intravenous diphenylhydantoin. Am Heart J 1967;74:410-2.

4. Chaikin P, Adir J. Unusual absorption profile of phenytoin in a massive overdose case. J Clin Pharmacol 1987;27:70-3.

5. Curtis DL, Piibe R, Ellenhorn MJ, Wasserberger J, Ordog G. Phenytoin toxicity: A review of 94 cases. Vet Hum Toxicol 1989;31:164-5. 
6. Wyte CD, Berk W. Severe oral phenytoin overdose dose not cause cardiovascular morbidity. Ann Emerg Med 1991;20:508-12.

7. Wit AL, Rosen MR, Hoffman BF. Electrophysiology and pharmacology of cardiac arrhythmias: Cardiac effects of diphenylhydantoin. Am Heart J 1975;90:397-404.

8. Brandolese R, Scordo MG, Spina E, Gusella M, Padrini R. Severe phenytoin intoxication in a subject homozygous for $\mathrm{CYP} 2 \mathrm{C} 9 * 3$. Clin Pharmacol Ther 2001;70:391-4.

9. Mirvis DM, Goldberger AL; Electrocardiography. In Braunwald Eugene, Zipes DP, Libby Peter (eds.): Heart disease. $6^{\text {th }}$ ed. Philadelphia, Pennsylvania: W.B. Saunders company; 2001, p. 119.
10. Barber MJ, Starmer CF, Grant AO. Blockade of cardiac sodium channels by amitriptyline and diphenylhydantoin. Evidence for two use-dependent binding sites. Circ Res 1991;69:677-96.

11. Evers ML, Izhar A, Aqil A. Cardiac monitoring after phenytoin overdose. Heart Lung 1997;26:325-8.

12. Kawasaki C, Nishi R, Uekihara S, Hayano S, Otagiri M. Charcoal hemoperfusion in the treatment of phenytoin overdose. Am J Kidney Dis 2000;35:323-6.

Accepted on 12-03-2009

Source of Support: Nil, Conflict of Interest: None declared. 